\title{
Enantioselective Synthesis of Some Nicotiana Alkaloids
}

\author{
Siavosh Mahboobi and Wolfgang Wiegrebe*) \\ Institute of Pharmacy, University of Regensburg, P.O. Box 397, \\ D-8400 Regensburg, Germany
}

Received July 17, 1987

A modified approach to myosmine (6) via a silyl enol ether of 3-acetylpyridine (1) is described. Chiral reduction of 6 with $N$-(benzyloxycarbonyl)- $L$ proline $/ \mathrm{NaBH}_{4}$ and formylation leads to $(R)-N$-formylnornicotine (8) $(35 \%$ ee) which in turn is converted to $(R)$-nornicotine (11) and $(R)$-nicotine (10).

\section{Enantioselektive Synthese einiger Nicotiana-Alkaloide}

Wir beschreiben eine modifizierte Synthese des Myosmins (6) über einen Silylenolether des 3-Acetylpyridins (1). Chirale Reduktion von 6 mit NBenzyloxycarbonyl-L-Prolin/ $\mathrm{NaBH}_{4}$ und $\mathrm{N}$-Formylierung ( $35 \%$ ee) führen zu (R)-N-Formylnornicotin (8), das in (R)-Nornicotin (11) bzw. (R)Nicotin (10) überführt wird.
Various methods for the synthesis of nicotiana alkaloids as racemates are reported, inter alia ${ }^{1-3)}$. We here describe a modified approach to myosmine (6) and strategies for chiral syntheses of nicotine derivatives.

Methyl-arylketones react with Böhme-Eschenmoser-salt ${ }^{4)}$ to the pertinent Mannich bases (type 4) which in turn are converted to the 3-oxo-3-arylbutyronitriles (type 5) ${ }^{3}$. Partial hydrogenation with concomittant ring closure leads to 2aryl-1-pyrrolines (type 6). This method gives very low yields with methyl-arylketones having low $\mathrm{C}-\mathrm{H}$-acidity of the methyl group as we recognized in our synthesis of Preininger's alkaloid $^{5)}$. So we adapted Danishefs $k y$ 's idea of activating the methyl group ${ }^{6)}$ via its pertinent silyl enol ether ${ }^{6)}$ : 3-acetylpyridine $^{7)}$ (1) was silylated in $95 \%$ yield with $\mathrm{F}_{3} \mathrm{C}-$ $\mathrm{SO}_{2}-\mathrm{O}-\mathrm{Si}\left(\mathrm{CH}_{3}\right)_{3}{ }^{8)}$ to $2 ; 2$ reacted with dimethyl-methylenimmonium iodide to give 3 , which was hydrolyzed to the Mannich base 4. 4-HCl was treated with $\mathrm{CN}^{-}$to afford the<smiles>CC(=O)c1cccnc1</smiles>

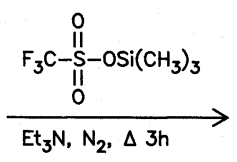

1<smiles>C[NH+](C)CCC(=O)c1cccnc1</smiles>

4

$\downarrow \begin{aligned} & \mathrm{KCN} \\ & \mathrm{H}_{2} \mathrm{O}, 40^{\circ}\end{aligned}$<smiles>N#CCCC(=O)c1cccnc1</smiles><smiles>C=C(c1cccnc1)c1cccnc1</smiles>

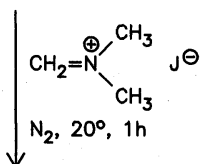<smiles>c1cncc(C2=NCCC2)c1</smiles>

nitrile 5 , the overall yield 2 to 5 is $72-75 \%$. Partial hydrogenation of 5 with Raney-Ni in $\mathrm{EtOH} / \mathrm{NH}_{3}$ led to myosmine (6). An exceeding hydrogenation to racem. nornicotine ${ }^{3)}$ is prevented by our conditions (cf. Experim. Part) (Scheme 1).

Chiral reduction generates a centre of chirality at $\mathrm{C}-1$ of the former pyrroline group.

Chiral reductions of imines being part of indol and isoquinoline alkaloids with Iwakuma's reagent ${ }^{9)}$ are known. In our hands usual cleavage of the $\mathrm{N}$-borane adduct 7 as described ${ }^{9)}$ does not give any defined product. Therefore, we used our work-up procedure with simultaneous $\mathrm{N}$-acylation ${ }^{4,10)}$, leading to the rotamers of (+)-(R)-N-formylnornicotine (8) in $35 \%$ ee and $90 \%$ chemical yield. Routine procedures ${ }^{1)}$ (Scheme 2) give rise to (+)-(R)-nicotine (10) and (+)-(R)-nornicotine (11) of equal optical purity (Scheme 2 ).
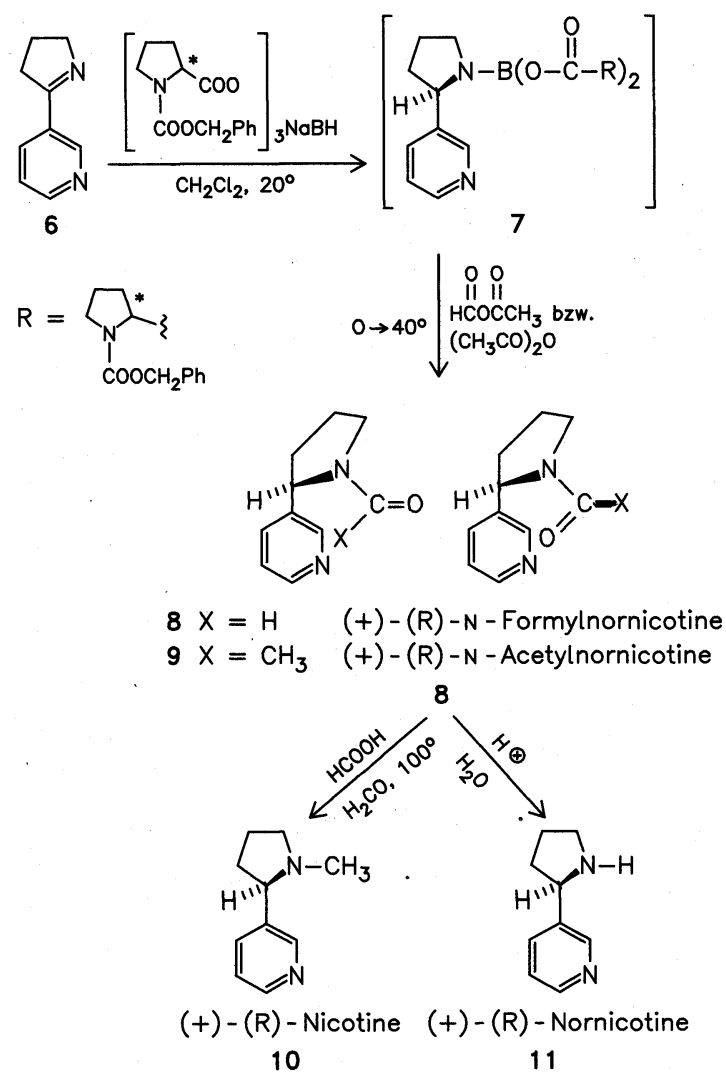
Use of acetic anhydride instead of the mixed anhydride $\mathrm{H}-\mathrm{CO}-\mathrm{O}-\mathrm{CO}-\mathrm{CH}_{3}$ during work-up after chiral reduction affords the racemate of $\mathrm{N}$-acetyl-nornicotine (9) ${ }^{111}$ with the enantiomer $\mathbf{R}-\mathbf{9}$ being enriched.

\section{Experimental part}

General remarks: lit ${ }^{10)}$. - Kugelrohr distillations were performed in a Büchi apparatus with at least 5 bulbs and twofold cooling with dry ice. The external temp. is cited.

\section{1-Trimethylsilyloxy-1-(3-pyridyl)-ethene (2)}

To $6.05(0.05 \mathrm{~mol}) 3$-acetylpyridine (1) ${ }^{7)}$ in $80 \mathrm{ml}$ absol. benzene and $6 \mathrm{~g}$ $\mathrm{Et}_{3} \mathrm{~N}$ were added drop by drop $23.05 \mathrm{~g} \mathrm{~F}_{3} \mathrm{C}-\mathrm{SO}_{2}-\mathrm{O}-\mathrm{Si}\left(\mathrm{CH}_{3}\right)_{3}{ }_{3}{ }^{8}$ then the mixture was refluxed for $3 \mathrm{~h}$. The upper phase (benzene) was evaporated $i$. vac. and the residue sublimated under the condition of Kugelrohr distillation $\left(50-60^{\circ} \mathrm{C}, 0.05 \mathrm{~mm} \mathrm{Hg}\right)$ : White crystals $(9.18 \mathrm{~g}, 95 \%)$, m. p. 52-53 ${ }^{\circ} \mathrm{C} .-{ }^{1} \mathrm{H}-\mathrm{NMR}: \delta(\mathrm{ppm})=0.35\left(\mathrm{~s} ; 9 \mathrm{H}, 3 \times \mathrm{CH}_{3}\right), 4.78(\mathrm{~d} ; \mathrm{J}=3.3$ $\left.\mathrm{Hz}, 1 \mathrm{H}, \mathrm{C}=\mathrm{CH}_{2}\right), 5.24\left(\mathrm{~d} ; \mathrm{J}=3.3 \mathrm{~Hz}, 1 \mathrm{H}, \mathrm{C}=\mathrm{CH}_{2}\right), 7.90-8.13(\mathrm{~m} ; 1 \mathrm{H}$, aromat.), 8.53-8.71 (m; 1H, aromat.), 8.80-9.16 (m;2H, aromat.).

\section{3-Cyano-1-(3-pyridyl)-propan-1-one (5)}

$7.73 \mathrm{~g}(0.04 \mathrm{~mol}) 2$ were dissolved in $20 \mathrm{ml}$ absol. $\mathrm{CH}_{2} \mathrm{Cl}_{2}$ under purified $\mathrm{N}_{2}$. Then $8.14 \mathrm{~g} \mathrm{(10 \%} \mathrm{excess)} \mathrm{dimethyl-methylenimmonium} \mathrm{iodide}{ }^{4)}$ were added at $0{ }^{\circ} \mathrm{C}$ in one portion under purified $\mathrm{N}_{2}$. After addition cooling is removed and after $1 \mathrm{~h} \mathrm{CH}_{2} \mathrm{Cl}_{2}$ is evaporated $i$. vac. under $\mathrm{N}_{2}$. The residue (colourless oil 3; the structure of $\mathbf{3}$ is deduced from Danishefsky's publication $^{6)}$ ) was dissolved at $0^{\circ} \mathrm{C}$ in $2 \mathrm{~N} \mathrm{HCl}$, excess $\mathrm{HCl}$ was evaporated $i$. vac. leaving a colourless oil 4 . In the hood dry $\mathrm{KCN}(3.90 \mathrm{~g}, 50 \% \mathrm{ex}-$ cess) was added in one portion under $\mathrm{N}_{2}$ followed by $400 \mathrm{ml}$ water of $40{ }^{\circ} \mathrm{C}$. After stirring under $\mathrm{N}_{2}$ at this temp. until 4 had disappeared (tlc control; $\mathrm{Al}_{2} \mathrm{O}_{3} /$ ethyl acetate or $\mathrm{SiO}_{2}$ /ethyl acetate) the mixture was cooled to room temp. and extracted with $\mathrm{CH}_{2} \mathrm{Cl}_{2}$. Column chromatography (CC) $\left(\mathrm{Al}_{2} \mathrm{O}_{3}\right.$ /ethyl acetate) afforded $4.60 \mathrm{~g}(72 \%) 5 ; \mathrm{m}$. p. $66^{\circ} \mathrm{C}\left(\mathrm{Et}_{2} \mathrm{O}\right.$, lit. $\left.{ }^{3)}: 66-67^{\circ} \mathrm{C}\right)$. - IR (KBr): $2264(\mathrm{C} \equiv \mathrm{N}), 1700 \mathrm{~cm}^{-1}(\mathrm{C}=\mathrm{O}) .-{ }^{1} \mathrm{H}-\mathrm{NMR}$ : $\left(\mathrm{CDCl}_{3}\right): \delta(\mathrm{ppm})=2.60-2.95\left(\mathrm{~m} ; \mathrm{J}=6 \mathrm{~Hz}, 2 \mathrm{H}, \mathrm{CH}_{2}\right), 3.30-3.55(\mathrm{~m} ; \mathrm{J}=$ $\left.6 \mathrm{~Hz}, 2 \mathrm{H}, \mathrm{CH}_{2}\right), 7.38-7.60$ (m; $1 \mathrm{H}$, aromat.), 8.15-8.38 (m; $1 \mathrm{H}$, aromat.) 8.80-8.98 (m;1H, aromat.), 9.16-9.30 (m; $1 \mathrm{H}$, aromat.).

\section{Myosmine (6)}

Contrary to Leete $^{3)}$ we hydrogenated 5 at atmospheric pressure and $40-50^{\circ} \mathrm{C}$. - To $2.92(20 \mathrm{mmol}) 5 \mathrm{in} 100 \mathrm{ml}$ absol. EtOH were added $3 \mathrm{ml}$ of absol. EtOH which was saturated with $\mathrm{NH}_{3}$ at $0{ }^{\circ} \mathrm{C}$, and $1.5 \mathrm{~g}$ Raney$\mathrm{Ni}$. - The reaction was controled by tlc $\left(\mathrm{Al}_{2} \mathrm{O}_{3}\right.$ /ethyl acetate or $\mathrm{Al}_{2} \mathrm{O}_{3} /$ $\mathrm{Et}_{2} \mathrm{O}$ ) in order to prevent further reduction of the $\mathrm{C}=\mathrm{N}$ double bond. When 5 had been converted completely, the mixture was filtered and evaporated and the residue was purified (short column; $\mathrm{Al}_{2} \mathrm{O}_{3}$ /ethyl acetate) and distilled (Kugelrohr, $60^{\circ} \mathrm{C}, 0.05 \mathrm{~mm} \mathrm{Hg}$ ): $2.26 \mathrm{~g}$ (85\%) white crystals, m. p. $43-43.5^{\circ} \mathrm{C}$ (lit. ${ }^{12)}$ : $40.5-42{ }^{\circ} \mathrm{C}$ ), picrate: m. p. $183.5-185^{\circ} \mathrm{C}$ (lit. $\left.)^{3}: 183-185^{\circ} \mathrm{C}\right) .-{ }^{1} \mathrm{H}-\mathrm{NMR}$ of 6 (base): $\delta(\mathrm{ppm})=1.85-2.26(\mathrm{~m} ; 2 \mathrm{H}$, pyrroline), 2.80-3.13 (m;2H, pyrroline), 3.95-4.26 (m; $2 \mathrm{H}$, pyrroline), 7.25-7.5 (m; 1H, aromat.), 8.10-8.34 (m; $1 \mathrm{H}$, aromat.), 8.54-8.8 (m; $1 \mathrm{H}$, aromat.), 8.96-9.13 (m; 1H, aromat.).

\section{$(+)-(R)-N$-Formylnornicotine (8) and (+)-(R)-N-Acetylnornicotine (9)}

Modifying Iwakuma's procedure his reagent was prepared as follows: $1.98 \mathrm{~g}$ (7.98 mmol) $\mathrm{N}$-benzyloxycarbonyl-L-proline were added in portions to a suspension of $79.44 \mathrm{mg}(2.1 \mathrm{mmol}) \mathrm{NaBH}_{4}$ in $13 \mathrm{ml}$ absol. THF at $5{ }^{\circ} \mathrm{C}$ under $\mathrm{N}_{2}$ and stirring. Stirring at this temp. was continued until development of $\mathrm{H}_{2}$ had ceased. After $3 \mathrm{~h}$ at room temp. THF was evaporated at $10^{\circ} \mathrm{C}$ under $\mathrm{N}_{2}$, the residue was dried at $20^{\circ} \mathrm{C}, 0.05 \mathrm{mmg} \mathrm{Hg}$, and dissolved in $4 \mathrm{ml}$ absol. $\mathrm{CH}_{2} \mathrm{Cl}_{2}$ at $0^{\circ} \mathrm{C}$ under $\mathrm{N}_{2}$. - To this solution $219.3 \mathrm{mg}$
(1.5 mmol) 6 in $4 \mathrm{ml}$ absol. $\mathrm{CH}_{2} \mathrm{Cl}_{2}$ were added at $0{ }^{\circ} \mathrm{C}$ under $\mathrm{N}_{2}$. After $10 \mathrm{~h}$ at $0^{\circ} \mathrm{C}$ and stirring for $3 \mathrm{~d}$ at room temp. the solution was divided into two fractions (about 1.5 and $6.5 \mathrm{ml}$, respectively). Both solutions were evaporated separately (faint yellow oils). The major part was reacted at $0{ }^{\circ} \mathrm{C}$ with $6 \mathrm{ml}$ acetic formic anhydride, previously cooled to $0^{\circ} \mathrm{C}$. The mixture was stirred for $30 \mathrm{~min}$ at room temp. then $30 \mathrm{~min}$ at $40-50^{\circ} \mathrm{C}$, followed by evaporation of the excess of anhydride. To the residue was added $\mathrm{HClO}_{4}(70 \%)$ at $0{ }^{\circ} \mathrm{C}$. After $30 \mathrm{~min}$ at $0{ }^{\circ} \mathrm{C}$ and $30 \mathrm{~min}$ at room temp. the mixture was neutralized with $\mathrm{N} \mathrm{NaOH}$ at $0{ }^{\circ} \mathrm{C}$ and rapidly extracted with $\mathrm{CH}_{2} \mathrm{Cl}_{2}$. After drying and $\mathrm{CC}\left(\mathrm{Al}_{2} \mathrm{O}_{3} ; \mathrm{CH}_{2} \mathrm{Cl}_{2} / \mathrm{H}_{3} \mathrm{CCN}\right.$ 9:1) 8 was purified by Kugelrohr distillation $\left(95-100{ }^{\circ} \mathrm{C} ; 0.05 \mathrm{~mm} \mathrm{Hg}\right): 195.4 \mathrm{mg}(91 \%)$ colourless oil. - IR (film): $1665 \mathrm{~cm}^{-1}(\mathrm{CO}) .-{ }^{1} \mathrm{H}-\mathrm{NMR}: \delta(\mathrm{ppm})=$ 1.75-2.15 (m; 3H, pyrrolidine), 2.25-2.60 (m; $1 \mathrm{H}$, pyrrolidine); 3.47-3.97 (m; 2H, pyrrolidine), 4.85-5.20 (m; 1H, pyrrolidine), 7.27-7.54 (m; 2H, aromat.), 8.39 (s; $0.35 \mathrm{H}, \mathrm{N}-\mathrm{CH}=\mathrm{O}), 8.15$ (s; $0.65 \mathrm{H}$, $\mathrm{N}-\mathrm{CH}=\mathrm{O}), 8.67-8.40(\mathrm{~m} ; 2 \mathrm{H}$, aromat.). Because 8 was not separated on Chirasil $\left.{ }^{4}{ }^{10}\right)$ the ee was determined at the stage of $\mathrm{N}$-acetyl-nornicotine (9).

The minor fraction was processed analogously but instead of the mixed anhydride mentioned above $3 \mathrm{ml}$ acetic anhydride were used. Neutralization at $-5{ }^{\circ} \mathrm{C}$; $\mathrm{CC}$ with $\mathrm{CHCl}_{3}$; Kugelrohr distillation at $95^{\circ} \mathrm{C} / 0.05 \mathrm{~mm}$ $\mathrm{Hg}$ : $49.75 \mathrm{mg}(93 \%)$ colourless oil.

The ee was determined as described ${ }^{4},{ }^{10)}: 35.4 \%(+)-(\mathrm{R})-9$. - IR (film): $1660 \mathrm{~cm}^{-1}(\mathrm{CO}) .{ }^{1} \mathrm{H}-\mathrm{NMR}: \delta(\mathrm{ppm})=1.70-2.70(\mathrm{~m} ; 4 \mathrm{H}$, pyrrolidine), $1.82\left(\mathrm{~s} ; 3 \mathrm{H}, \mathrm{CO}-\mathrm{CH}_{3}\right), 3.48-3.85(\mathrm{~m} ; 2 \mathrm{H}$, pyrrolidine $), 4.83-5.28(\mathrm{~m}$; $1 \mathrm{H}$, pyrrolidine), $7.40-7.58(\mathrm{~m} ; 2 \mathrm{H}$, aromat.), $8.35-8.85(\mathrm{~m} ; 2 \mathrm{H}$, aromat). - The $\mathrm{ms}$ revealed the fragment ions described ${ }^{11)}$ in similar rel. int.

\section{$(+)-(R)$-Nicotine (10)}

$88 \mathrm{mg}(0.5 \mathrm{mmol}) 8$ were stirred in an autoklave with $2 \mathrm{ml} \mathrm{HCOOH}$ (98\%) and $2 \mathrm{ml} \mathrm{H}_{2} \mathrm{CO}(37 \%)$ at $100^{\circ} \mathrm{C}$ for $18 \mathrm{~h}$. After cooling to $0{ }^{\circ} \mathrm{C}$ the excess of the reagent was evaporated at $28^{\circ} \mathrm{C}$, the residue (oil) was triturated at $0{ }^{\circ} \mathrm{C}$ with pre-cooled $2 \mathrm{~N} \mathrm{NaOH}$, the mixture was saturated with $\mathrm{NaCl}$ and extracted with $\mathrm{CH}_{2} \mathrm{Cl}_{2}$ repeatedly. After drying $\left(\mathrm{Na}_{2} \mathrm{SO}_{4}\right)$ and Kugelrohr distillation with intensive cooling $\left(35-40{ }^{\circ} \mathrm{C} ; 0.01 \mathrm{~mm} \mathrm{Hg}\right.$, Lit. ${ }^{13)}: 109^{\circ} \mathrm{C}, 8 \mathrm{~mm} \mathrm{Hg}$ ) we obtained 10 as a colourless oil $(81 \mathrm{mg}, 91 \%)$. - IR (film): $2793 \mathrm{~cm}^{-1}\left(\mathrm{~N}-\mathrm{CH}_{3}\right) .-{ }^{1} \mathrm{H}-\mathrm{NMR}: \delta(\mathrm{ppm})=1.50-2.52(\mathrm{~m}$; $5 \mathrm{H}$, pyrrolidine), $2.11\left(\mathrm{~s} ; 3 \mathrm{H}, \mathrm{N}-\mathrm{CH}_{3}\right), 2.92-3.44(\mathrm{~m} ; 2 \mathrm{H}$, pyrrolidine), 7.12-7.37 (m; 1H, aromat.), 7.59-7.80 (m; 1H, aromat.), 8.38-8.63 (m; $2 \mathrm{H}$, aromat.). $-[\alpha]_{\mathrm{D}}^{24}=+28.07^{\circ}$ (aqueous $\left.1 \% \mathrm{KOH}^{13)}, \mathrm{c}=0.9\right): 36.1 \%$ ee $\left(\right.$ lit: $\left.{ }^{13)}[\alpha]_{\mathrm{B}}^{24}=+77.78^{\circ}\right)$.

\section{$(+)-(R)$-nornicotine (11)}

$52.86 \mathrm{mg}(0.3 \mathrm{mmol}) 8$ were refluxed with $10 \mathrm{ml} 3 \mathrm{~N} \mathrm{HCl}$ for $2 \mathrm{~h}$, then cooled to $0^{\circ} \mathrm{C}$ and made alcaline with $\mathrm{NaHCO}_{3}$. After saturation with $\mathrm{NaCl}$ and extraction with $\mathrm{CH}_{2} \mathrm{Cl}_{2}$ the org. layer was dried $\left(\mathrm{Na}_{2} \mathrm{SO}_{4}\right)$ and carefully evaporated $\left(20^{\circ} \mathrm{C}, 20 \mathrm{~mm} \mathrm{Hg}\right)$ in order to prevent evaporation of 11 : colourless pure residue (NMR). Careful Kugelrohr distillation at $25-30^{\circ} \mathrm{C}, 0.2 \mathrm{mg} \mathrm{Hg}\left(118-119^{\circ} \mathrm{C}, 3 \mathrm{~mm} \mathrm{Hg}{ }^{14}\right)$ afforded $40 \mathrm{mg}(90 \%)$ 11 as an colourless oil. - IR (film): $3300 \mathrm{~cm}^{-1}$ (br., NH). ${ }^{1} \mathrm{H}-\mathrm{NMR}$ : $\delta(\mathrm{ppm})=1.50-2.40(\mathrm{~m} ; 4 \mathrm{H}$, pyrrolidine $), 1.98\left(\mathrm{~s} ; 1 \mathrm{H}, \mathrm{NH}, \mathrm{D}_{2} \mathrm{O}\right.$ exchan ge), 2.83-3.42 ( $\mathrm{m} ; 2 \mathrm{H}$, pyrrolidine), 4.04-4.35 ( $\mathrm{m} ; 1 \mathrm{H}$, pyrrolidine), 7.13-7.40 (m; 1H, aromat.), 7.60-7.81 (m; 1H, aromat.), 8.40-8.68 (m; $2 \mathrm{H}$, aromat.).

\section{References}

1 M. Nakane and C. R. Hutchinson, J. org. Chem. 43, 3922 (1978).

2 G. F. Alberici, J. Andrieux, G. Adam, and M. M. Plat, Tetrahedron Lett. 1983, 1937.

3 E. Leete, M. R. Chedekel, and G. B. Bodem, J. Org. Chem. 37, 4465 (1972). 
4 H. Brunner, R. Becker, S. Mahboobi, and W. Wiegrebe, Angew. Chem. 97, 969 (1985) and lit. cited there.

5 S. Mahboobi and W. Wiegrebe, Sci. Pharm. 54, 217 (1986).

6 S. Danishefski, T. Kitahara, R. McKee, and P. F. Schuda, J. Am. Chem. Soc. 98, 6715 (1976).

7 3-Acetylpyridine from EGA-Chemie, D-7900 Steinheim.

8 G. Simchen and W. Kober, Synthesis 1976, 259.

9 K. Yamada, M. Takeda, and T. Iwakuma, J. Chem. Soc. Perkin Trans. I 1983, 265.
10 H. Brunner, A. Kürzinger, S. Mahboobi, and W. Wiegrebe, Arch. Pharm. (Weinheim) 321, 73 (1988).

11 A. H. Warfield, W. D. Galloway, and A. G. Kallianos, Phytochemistry 11,3371 (1972).

12 D. Spitzner, Synthesis 1977, 242.

13 E. Späth, C. S. Hicks, and E. Zajic, Ber. Dtsch. Chem. Ges. 68, 1388 (1935).

14 T. Kisaki and E. Tamaki, Arch. Biochem. Biophys. 92, 351 (1961).

[Ph 371] 